\title{
Electronic communication between providers of primary and secondary care
}

\author{
P J Branger, J C van der Wouden, B R Schudel, E Verboog, J S Duisterhout, J van der Lei, \\ $\mathrm{J} H$ van Bemmel
}

\begin{abstract}
Objective-To study the effects of the introduction of electronic data interchange between primary and secondary care providers on speed of communication, efficiency of data handling, and satisfaction of general practitioners with communication.

Design-Comparison of traditional paper based communication for laboratory reports and admission-discharge reports between hospital and general practitioners and electronic data interchange.

Setting-Twenty-seven general practitioners whose offices were equipped with a practice information system and two general hospitals.

Outcome measures-Paper based communication was evaluated by questionnaire responses from and interviews with care providers; electronic communication was evaluated by measuring time intervals between generation and delivery of messages and by assessing doctors' satisfaction with electronic data interchange by questionnaire.

Results-Via paper mail admission-discharge reports took a median of 2-4 days, and laboratory reports 2 days, to reach general practitioners. With electronic data interchange almost all admissiondischarge reports were available to general practitioners within one hour of generation. When samples were analysed on the day of collection (as was the case for 174/542 samples in one hospital and $443 / 854$ in the other) the laboratory reports were also available to the general practitioner the same day via electronic data interchange. Fifteen general practitioners (of the 24 who returned the questionnaire) reported that the use of electronic admissiondischarge reports provided more accurate and complete information about the care delivered to their patients. Ten general practitioners reported that electronic laboratory reports lessened the work of processing the data.
\end{abstract}

Conclusion-Electronic communication between primary and secondary care providers is a feasible option for improving communication.

\section{Introduction}

In the Netherlands, as in the United Kingdom general practitioners function as gatekeepers between primary and secondary care. Good communication between primary and secondary care providers is therefore essential for coordinating care for individual patients and providing continuity.

Previous research has highlighted the problems of communication between primary and secondary care providers. These studies have shown both the poor quality of information provided and the delays in receiving it. ${ }^{1-8}$ In particular, some of these delays jeopardise continuing care, especially in elderly patients.
New technologies are now emerging that have considerable potential for improving communication. One such technology is electronic data interchange, defined as "standard electronic messages conveyed from one computer to another without manual intervention." In the Netherlands several organisations have cooperated to standardise messages in health care using the Edifact standard, ${ }^{1111}$ which describes the syntax of messages. Several standardised messages are available, including a laboratory report and an admission-discharge report. Using such a standardised message a laboratory can, for instance, transmit test results electronically to a general practitioner's computer system, which can then manipulate and store the data automatically.

In the Netherlands the infrastructure required for electronic data interchange is emerging. Many hospitals have already had years of experience with information systems, and in primary care computer based patient records are gaining ground rapidly. At present half of all Dutch general practitioners (over 3000) are using an information system in daily practice, and 1300 of them are using computer based patient records.

In this paper we describe a project that studied electronic communication between hospitals and general practioners. The aims of the study were $(a)$ to introduce electronic data interchange in a health care environment, $(b)$ to assess the gain in speed of delivery of reports achieved using electronic data interchange instead of paper mail, $(c)$ to measure the influence of electronic data interchange on handling of data in daily practice, and (d) to evaluate its potential to support doctors in achieving continuing medical care.

\section{Methods}

In the city of Apeldoorn the communication project was started in 1988, with 27 general practitioners participating. Sixteen were single handed, eight worked in two person practices, and three worked in health centres and together they provided care for 50000 patients. The two regional general hospitals (hospital 1 and hospital 2) participated in the project. All general practitioners were using the information system Elias. ${ }^{12}$ In addition to financial and administrative functions Elias provides a computer based patient record that is used by the general practitioner to maintain patient data.

\section{ELECTRONIC COMMUNICATION}

In December 1989 three types of electronic messages were introduced: admission-discharge reports from hospital to general practice; laboratory reports from hospital to general practice; and free text messages between general practitioners. The free text messages were unstructured plain text, whereas the admissiondischarge reports and the laboratory reports were
Address for correspondence: Dr Branger.

$B M 7$ 1992;305:1068-1070 
TABLE I-Estimated time intervals for admission/discharge reports and laboratory reports when traditional mail delivery was used

\begin{tabular}{|c|c|c|c|c|c|c|c|c|}
\hline & \multicolumn{8}{|c|}{ No of GPs reporting an average time in days of: } \\
\hline & 1 & 2 & 3 & 4 & 5 & 6 & 7 & $\begin{array}{c}\text { No } \\
\text { answer }\end{array}$ \\
\hline \multicolumn{9}{|c|}{ Admission discharge reports } \\
\hline Hospital 1 & 1 & 10 & 6 & 1 & & & & 6 \\
\hline Hospital 2 & 4 & 3 & 2 & 2 & 5 & & 2 & 6 \\
\hline \multicolumn{9}{|c|}{ Laboratory reports } \\
\hline Hospital 1 & 3 & 11 & 6 & 3 & 1 & & & \\
\hline Hospital 2 & 7 & 11 & 5 & & 1 & & & \\
\hline
\end{tabular}

structured standardised messages. The general practitioner's information system used the admissiondischarge reports to create and update automatically an overview of admitted and discharged patients. ${ }^{13}$ The general practitioner could obtain a printout of this computer based overview, optionally combined with relevant data from the computer based patient record, to be used as a memorandum for home or hospital visits. After verification by the general practitioner, data from laboratory reports were stored automatically in the patient record.

We used a commercially available communication network, on which each hospital and general practice had its own electronic mail address. The costs of this network were an entrance fee of Dfl 100, a monthly subscription of Dfl 15, and a charge of Dfl 1.50 per message of 225 lines.

\section{EVALUATION}

The evaluation consisted of four phases.

Baseline study-Before the introduction of electronic data interchange we sent general practitioners a questionnaire that elicited practice characteristics, how long admission-discharge reports and laboratory reports took to arrive by mail, and the amount of communication among general practitioners. We also conducted personal interviews at the two hospital patient administration departments and laboratories to assess the methods used to generate and send reports.

Message flow measurements-After electronic data interchange had been implemented we measured over 10 weeks (January-March 1990) the time between generation of all admission-discharge reports and all laboratory reports in the two hospitals and arrival of those messages at the general practitioners' offices. During the last three weeks of the study period we also measured the time between the arrival of the messages at the general practitioners' offices and reading of the message by the general practitioners. To perform these measurements we added programs to the hospital computer system and to Elias that recorded generation and use of the message, and, after removing data that could identify patient or doctor, automatically sent a copy of the message and the measurements to our research computer. We could not perform measurements on free text messages because it was impossible to eliminate the risk of violating privacy. At this time 21 general practitioners had started using electronic data interchange. The other general practitioners started two to four weeks later so they did not participate in this part of the study.

Efficiency study-We evaluated whether electronic data interchange increased the percentage of laboratory test results stored in Elias by the general practitioner and whether it reduced transcription errors. For three months before and three months after the introduction of electronic data exchange we collected laboratory test results from the hospital laboratory system and compared these data with the data held in the computer based patient records of seven general practitioners.

User satisfaction study-To assess the level of user acceptance we sent general practitioners a questionnaire after they had had three months' experience with electronic data interchange. We asked them whether electronic admission-discharge reports provided more accurate information on the care being delivered to their patients than paper reports; whether electronic laboratory reports demanded less work to process than paper reports; and whether the use of electronic mail for exchanging patient data had improved communication between general practitioners.

\section{Results}

BASELINE STUDY

Three times a week hospital 1 produced for each general practitioner an admission-discharge report containing a list of admitted and discharged patients. This list was then mailed to the general practitioners. In hospital 2 each general practitioner had a mailbox, in which a copy of the patient's identity card was placed at the time of admission or discharge; the general practitioner emptied this mailbox whenever he or she was in the hospital. The median time between the admission or discharge of a patient and delivery of the report at the general practitioner's office, as estimated by the 24 general practitioners who returned the questionnaire, was two days for hospital 1 and four days for hospital 2 (table I).

At both hospital laboratories sample taking and analysis were done throughout the day, whereas laboratory reports were generated only once a day (at about $400 \mathrm{pm}$ ). Results of emergency tests ordered by general practitioners were reported by telephone as soon as they became available. Sometimes general practitioners had to wait three days or longer for nonemergency results because the test was not performed every day-for example, thyroid function tests were performed only once a week; the sample was obtained shortly before the weekend and was reported on Monday; or the test was performed at a specialist laboratory at a different location. The median time between printing of the laboratory report and delivery of the report to the general practitioner's office, as estimated by the general practitioners, was two days for both hospitals (table I).

Communication between general practitioners about patients occurred most often when, during a night or weekend shift, one general practitioner saw another general practitioner's patient; the first would usually write a note describing this consultation and would put the note in the mailbox at hospital 2 or at the office of the general practitioner. In some cases the telephone was used.

\section{MESSAGE FLOW MEASUREMENTS}

During the 10 week period 1388 admission-discharge reports and 1396 laboratory reports were sent electronically. Admission-discharge reports were generated and transmitted twice a day and laboratory reports once a day. Almost all electronic admission-discharge reports were available to the general practitioners within one hour of generation (table II). Of all electronic laboratory reports, $174 / 542(32 \%)$ from hospital 1 and 443/854 (52\%) from hospital 2 were available to the general practitioners on the day that the samples were collected (table III). The remaining reports arrived later because analysis of the samples was delayed.

The median time between the messages becoming available to the general practitioners and the general practitioners reading them was $0-4$ hours for five general practitioners, 4-8 hours for four general practitioners, 8-24 hours for five general practitioners, 2448 hours for four general practitioners, and more than 48 hours for three general practitioners. The time measurements were not adjusted for off duty hours and 
holidays, during which general practitioners did not work with Elias.

EFFICIENCY STUDY

All 7 general practitioners stored more test results with than without electronic data interchange (table IV). We checked for errors 3635 test results entered in Elias manually and 5633 test results entered electronically. Among the results entered manually we found 19 errors $(0.5 \%)$; in those entered electronically we found none.

TABLE IV-Numbers of laboratory tests requested and stored in the computer based patient record by individual general practitioners without and with electronic data interchange

\begin{tabular}{lccccr}
\hline & \multicolumn{2}{c}{$\begin{array}{c}\text { Without electronic data } \\
\text { interchange }\end{array}$} & & \multicolumn{2}{c}{$\begin{array}{c}\text { With electronic data } \\
\text { interchange }\end{array}$} \\
\cline { 2 - 3 } \cline { 5 - 6 } GP & $\begin{array}{c}\text { No } \\
\text { requested }\end{array}$ & $\begin{array}{c}\text { No (\%) } \\
\text { stored }\end{array}$ & & $\begin{array}{c}\text { No } \\
\text { requested }\end{array}$ & $\begin{array}{c}\text { No (\%) } \\
\text { stored }\end{array}$ \\
\hline A & 1732 & $1139(66)$ & & 1775 & $1266(71)$ \\
B & 1485 & $1324(89)$ & & 1389 & $1286(93)$ \\
C+D & 3400 & $2239(67)$ & & 4396 & $3191(73)$ \\
E & 735 & $616(84)$ & & 1061 & $897(85)$ \\
F & 2030 & $1515(75)$ & & 2003 & $1506(75)$ \\
G & 622 & $244(39)$ & & 552 & $384(70)$ \\
\hline
\end{tabular}

USER SATISFACTION

Of the 27 general practitioners, 23 returned the questionnaire on user experience with electronic data interchange. All except one general practitioner used free text messages for exchanging patient information. When asked to rate the benefits of this type of message on a scale of 0 (useless) to 5 (very useful), 16 general practitioners scored 5 , four 4 , one 3 , and two 2 . Fifteen general practitioners reported that use of electronic admission-discharge reports had provided more accurate knowledge on the care being delivered to individual patients. For the benefits of this type of message, 5 general practitioners scored 5 , ten 4 , four 3 , three 2 , and two 1 .

The use of electronic laboratory reports has two possible benefits: increased speed of reporting and integration of tests in the computer based patient record. For speed of reporting, six general practitioners scored 5 , three 4 , nine 3 , four 2 , and one 1 . Integration of tests into the computer based patient record, however, was valued much higher: 17 general practitioners scored 5 and six 4 . Ten general practitioners reported a decrease in workload.

\section{Discussion}

This first attempt to introduce electronic data interchange in Dutch health care was successful. Electronic communication between hospitals and general practitioners led to shorter delays in transmission of admission-discharge and laboratory reports to the general practitioners; it improved the amount of information recorded in general practitioners' computer based patient records and its accuracy; and most general practitioners were satisfied with these results. The network has remained in operation after the completion of the study period, and the general practitioners have integrated electronic communication into their daily practice. Thirty four general practitioners in Apeldoorn now use electronic communication.

The aim of this study was to test the feasibility of electronic communication in Dutch health care in a representative health care region. We therefore designed the electronic communication system so that it would not depend on any local factors and used a nationally available communication network and standardised messages.

Our results suggest that using electronic data interchange creates new possibilities for improving com- munication: admission-discharge reports are now issued twice a day, and general practitioners thus have an up to date overview of their patients in hospital. Most of the general practitioners judged that their knowledge about the care being delivered to individual patients had increased with the use of electronic data interchange. This contrasts with the situation with paper based communications. Several studies have found poor quality information transmitted between general practitioners and hospitals ${ }^{125}$ and delays in sending it.' Mageean, for example, found that half of discharged patients had contacted their general practitioner before the general practitioner had received any information from the hospital. ${ }^{4}$ Doeleman has argued that improved communication leads to improved cooperation and quality of care. ${ }^{8}$ We found that the general practitioners in our study greatly appreciated the use of free text messages for exchanging information on patients, especially for reporting patient encounters during night and weekend shifts.

The use of electronic data interchange for integrating laboratory test results into the computer based patient record led to a decreased workload for 10 out of 23 general practitioners. When test results are entered manually into the Elias system the program performs validity checks, thus presumably preventing most transcription errors. Even so we found that $0.5 \%$ of the test values entered manually into the computer based record were wrong. When the test results were entered electronically no such errors were observed.

We conclude that electronic data interchange has the potential to increase the efficiency of processing of information and to support continuity of medical care provided by general practitioners. Ament and L'Ortye report that a Dutch hospital spends Dfl 1000 to Dfl 1500 ( $£ 300$ to $£ 500$ ) on communication per general practitioner per year and conclude that electronic communication will lead to substantial cost savings in hospitals. ${ }^{1+}$ General practitioners on the other hand, must pay for communication they previously got for free. In Apeldoorn the users of the communication network share the costs. How in the future people will pay for widespread use of electronic communication, however, is an issue to be negotiated by hospitals, care providers, and health insurance companies. This financial issue must be clarified before large scale introduction of electronic communication is possible.

1 Tulloch AJ, Fowler GH, McMullan JJ, Spence JM. Hospital discharge reports: content and design. $B M 7$ 1975;4:443-6.

Hull FM, W'esterman RF. Referral to medical outpatients departments at teaching hospitals in Birmingham and Amsterdam. BMJ 1986;293:311-4.

3 Westerman RF, Hull FM, Bezemer PD, Gort G. A study of communication between general practitioners and specialists. Br $\mathcal{F}$ General Practice 1990;40: 445-9.

4 Mageean RJ. Study of "discharge communications" from hospital. BMf 1986:293:1283-5.

5 Williams EI, Fitton F. General practitioner response to elderly patients discharged from hospital. BM7 1990;300:159-61.

6 Jacobs IGH, Pringle MA Referral letters and replies from orthopaedic departments: opportunities missed. BM7 1990;301:470-3.

7 Sandler DA, Mitchell JRA. Interim discharge summaries: How are they bes delivered to general practitioners? BMF 1987;295:1523-5.

8 Doeleman F. Improving communication between general practitioners and specialists. Fam Pract 1987;4:176-82

9 Walker RJ, ed. First steps in EDI: Proceedings of COMPAT '89. Munich Blenheim Online, 1989

10 Electronic data imterchange for administration, commerce and transpon (EDIFACT): application level syntax rules. Geneva: United Nations Economic Commission for Europe, Trade Division, 1988. (ISO 9735.)

11 Kinkhorst OM, Hasman A, Van Kesteren ACA. Standardization of datainterchange in health care. In: Duisterhout JS, Hasman A, Salamon R, eds Telematics in medicine. Amsterdam: Elsevier Science, 1991:313-26.

12 Westerhof HP, Boon WM, Cromme PVM, van Bemmel JH. ELIAS, suppor for the Dutch general practitioner. In: Reichertz PL, Engelbrecht $R$ Piccolo $\mathrm{U}$, eds. Lecture notes in medical infornatics: present status of computer Piccolo L, eds. Lecture notes in medical informatics: present stans

13 Duisterhout JS, Branger PJ. EDIFACT message handling and integration into applications in the COPA project. In: Duisterhout JS, Hasman A, Salamo $\mathrm{R}$, eds. Telimatics in medicine. Amsterdam: Elsevier Science, 1991:151-62.

14 Ament A, L'Ortye M. Economic evaluation of electronic communication in health care (3i-project). In: O'Moore R, Bengtsson S, Bryant JR, Bryden JS, eds. Lecture notes in medical informatics. Volume 40. Proceedings of Medical Informatics Europe 90. Heidelberg: Springer, 1990:130-4.

(Accepted 9 Septembir 1992) 